\title{
An underactuated parallel-link gripper for a multicopter capable of plane perching
}

\author{
Maozheng Xu, Taku Senoo and Takeshi Takaki
}

\begin{abstract}
The need for a perching robot is increasing in the field of rescue and transportation. Accordingly studies on perching an object by attaching a robot arm to a perching robot have been conducted. However, almost all the studies related to perching have been conducted using an actuated or electric device. However, perching by using an electric device has several disadvantages, such as additional power consumption and an increase in the mass of the multicopter used to load the electric source. Instead of using an electric device, perching by using an underactuated gripper can effectively avoid these disadvantages. Accordingly, we developed an underactuated passive gripper that has the advantage of nonconsumption of electric power for perching. A method to confirm the available range for stable perching is one of the problems of using an underactuated passive gripper. Therefore, in this study, we analyze a multicopter carrying an underactuated parallel-link passive gripper for available plane perching. To enable perching on planes with different thicknesses and being embedded at different depths, we summarize the available perching range and limitations based on the friction cone theory. Our conclusion is supported by both theoretical and experimental results.
\end{abstract}

Keywords: Perching, Multicopter, Self-gravity, Parallel linkage, Underactuated gripper

\section{Introduction}

Recently, there has been an increase in the use of multicopters for rescue and transportation [1], and environmental monitoring $[2,3]$, and for express home delivery, whereby a robot arm is attached to the multicopter [4]. Express delivery using a multicopter, in response to people's consumption requirements, has several advantages. Therefore, studies need to be conducted on multicopters equipped with a mechanism for catching, and more importantly, perching.

The word perching was originally used to describe bird landing behavior. Extended to multicopter behavior, the term indicates a process in which the multicopter flies to land or sit on targets, such as pipes, sticks, or branches. However, the multicopter requires a certain mechanical device to execute the maneuver for perching on targets.

*Correspondence: takaki@hiroshima-u.ac.jp

Hiroshima University, Hiroshima, 1-4-1 Kagamiyama, Higashi-Hiroshima, 739-8527 Hiroshima, Japan
The design of the device depends on the shape and properties of the target. For example, for the multicopter to perch on a branch or cylindrical target, the gripper must be designed with birdlike talons; to perch on horizontal and vertical platforms, the gripper must be designed so that it can grasp the target firmly, without sliding down. In general, studies on perching can be divided into two categories: those on the control of perching and those on the mechanical structure of perching. In a previous study on perching control, researchers from New Mexico State University and Shanghai University developed bio-inspired trajectory generation for unmanned aerial vehicle (UAV) perching based on the tau theory $[5,6]$. A number of other researchers have focused on the mechanical structures of perching. These mechanical structures include grippers $[7,8]$, robot hands $[9,10]$, landing legs $[11,12]$, and other advanced mechanisms for grasping and perching. For example, researchers at the University of Twente and Aalborg University developed a mechanism that facilitates the reliable perching of UAVs 
on smooth vertical surfaces using a lightweight passive vacuum-cup technology and the absorption of aerial impacts [13].

However, the aforementioned mechanical structures for perching are actuated because they carry power supply devices. This increases the mass of the entire aerial system, which may result in an overload of the multicopter when flying or perching. Thus, studies on perching via passive mechanisms have been conducted to solve this problem. Researchers at the University of Utah developed an avian-inspired passive mechanism for quadrotor perching $[14,15]$. In this study, the proposed passive mechanism is capable of perching on a pipe-like object. Although this avian-inspired device can perch on a cylindrical object, it is composed of numerous complex mechanical structures. For the purpose of delivery or security monitoring, the target of perching often involves a man-made object, such as a planar structure, which only needs a simpler mechanism. Therefore, to achieve this purpose, we proposed an underactuated device with a simpler mechanical structure, which is shown in Fig. 1.

We successfully developed this underactuated parallellink gripper in an earlier study [16]; however, there was an insufficient discussion on perching. For example, to simplify the calculation, we assumed that the contact point of the upper side of the gripper was at the innermost position. However, in the real world, we must consider cases where the contact point of the gripper is at an arbitrary position, which is one of the focus areas of this study.

The remainder of the paper is organized as follows: the perching motion of the gripper device is described

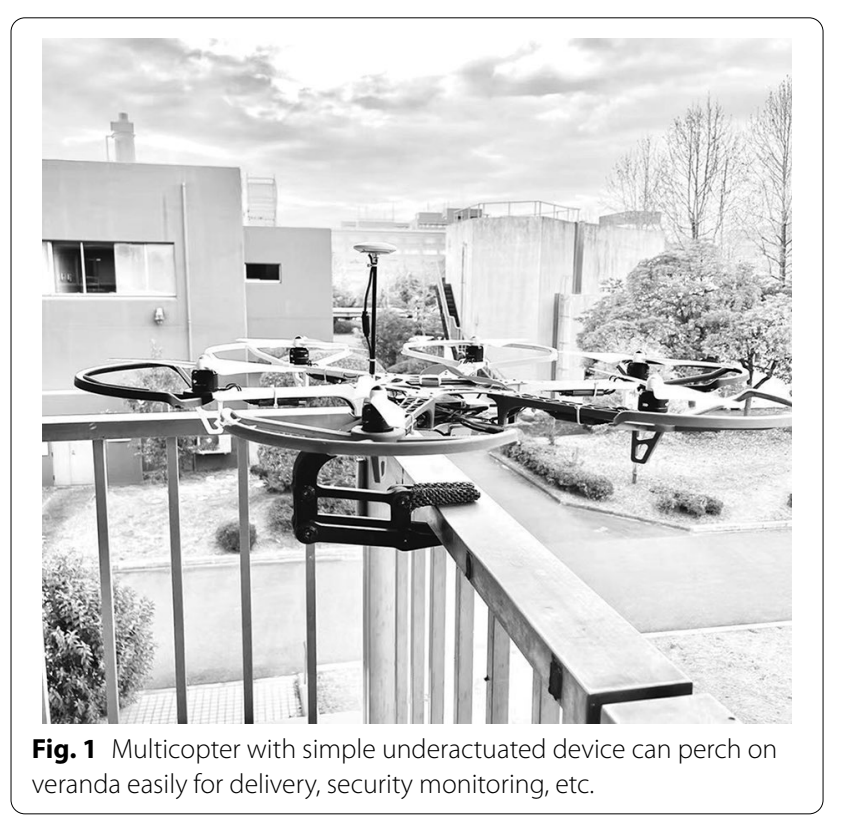

in "Perching motion of the gripper device" Section. The theoretical basis for the existing perching judgment is described in "Available condition for stably perching" Section, and subsequently, a static model for analysis is established. "Perching state judgment" Section focuses on the perching state judgment using the friction cone theory. "Parallel-link passive gripper prototype and mechanism integration" Section provides an overview of the perching device and mechanism integration. "Experiment" Section describes the experiment and a comparison of the theoretical and experimental results. Finally, "Conclusion" Section concludes this paper and presents a description of our future work.

\section{Perching motion of the gripper device}

A perching system can be divided into four parts, as shown in Fig. 2a. Link 1 is the assembling bar that connects the body of the multicopter and other parts of the gripper. Link 2, which is in the middle, is called the connecting bar, and its function is to connect the gripper and the assembling bar. Link 3 is the gripper, which has the function of grasping the upper side of the table. Link 4 is called the underside link bar, and it contacts the table and provides the grasping force for perching. Links 1-4 are composed of parallel devices, and as shown in Fig. 2a, the distance between each joint was assumed to be $l_{1}, l_{2}$, $l_{3}$, and $l_{4}$. Then, we can obtain the relationships $l_{1}=l_{3}$ and $l_{2}=l_{4}$.

Figure 2a-c describe the process of the perching motion, which consists of three stages. The first stage, shown in Fig. 2a, involves the approach of the multicopter. In this stage, the multicopter is set to land on the target and maintain a horizontal posture to perch on the target smoothly. Then, in the second stage of perching, the underside of Link 3 contacts the upper side of the target surface, as shown in Fig. 2b. Simultaneously, the motors of the multicopter stop running, the entire device declines because of its own gravity, and the front side of Link 4 rises until it touches the underside of the target. In the entire process, the body of the multicopter is maintained in a horizontal state, parallel to the target surface, so that the entire device is not tilted and maintains its balance (Fig. 2c).

\section{Available condition for stably perching}

In a previous study [16], we performed a preliminary analysis on a specific situation. In this case, the object on which the multicopter was to be perched was a table with a constant thickness. In addition, for the analysis and calculation, we assumed that the contact point $\boldsymbol{P}_{6}$ was at the innermost part of the gripper shown in Fig. 3. This could be considered a very special case. However, the generalizability of the model needs to be extended by varying the condition 


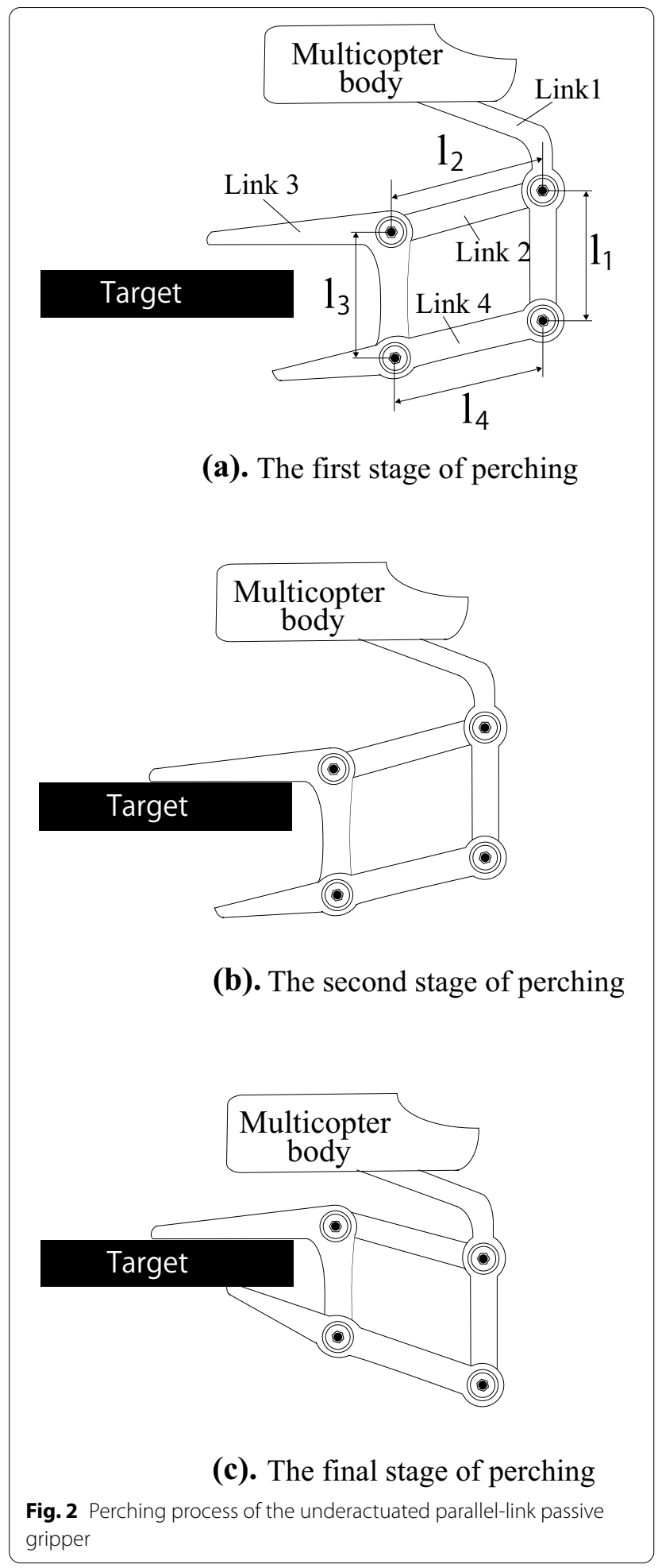

for practical applications. Thus, the main objective of this study is the analysis of perching success on a plane of varying thickness, embedded at an arbitrary depth (arbitrary contact point $\boldsymbol{P}_{6}$ ).

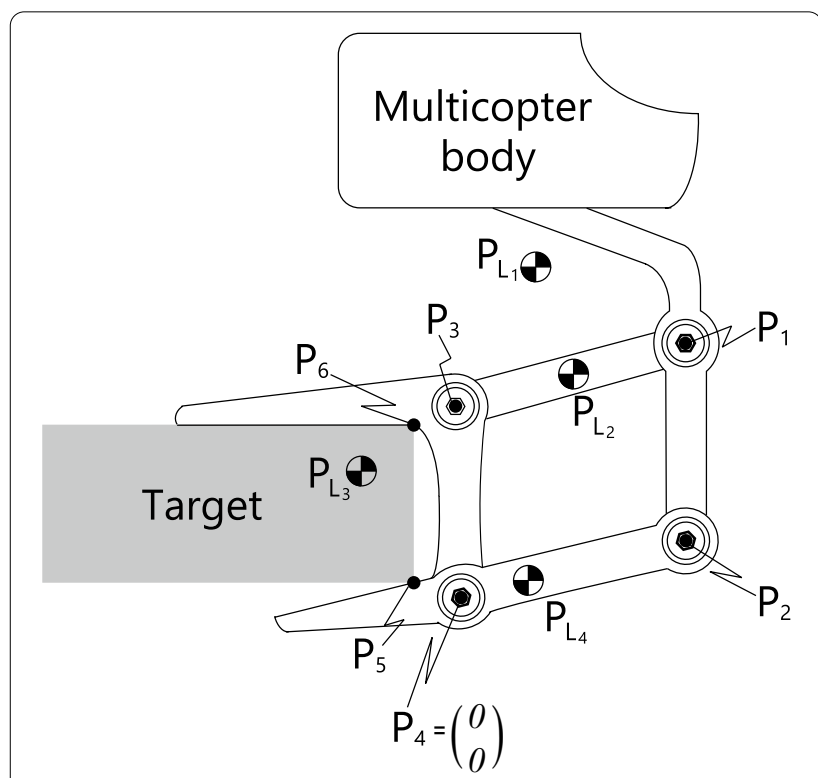

Fig. 3 Static model of the gripper device

\section{Anatomy of the static model}

As depicted in Fig. 3, the static model is established in 2 dimensions, and the entire device consists of four parts and six positions. Here, we assume that the gripper comes in contact with the target at points 5 and 6 , which are indicated by $P_{5}$ and $P_{6}$, respectively, as shown in Fig. 3. Based on this assumption, a component analysis can be performed for the entire device. We assume that $\boldsymbol{P}_{i}(i=1,2, \ldots, 6)$ and $\boldsymbol{F}_{i}(i=1,2, \ldots, 6)$ are the position and force vectors, respectively.

We define $\quad \boldsymbol{F}_{i}=\left[F_{i x}, F_{i y}\right]^{T}, \quad \boldsymbol{P}_{i}=\left[P_{i x}, P_{i y}\right]^{T}, \quad$ and $\boldsymbol{S}\left(\boldsymbol{P}_{i}\right)=\left[-P_{i y}, P_{i x}\right]$. Subsequently, the torque $\tau_{i}$ can be defined as follows:

$$
\tau_{i}=P_{i x} f_{i y}-P_{i y} f_{i x}=\left[-P_{i y}, P_{i x}\right]\left[\begin{array}{l}
f_{i x} \\
f_{i y}
\end{array}\right]=\boldsymbol{S}\left(\boldsymbol{P}_{i}\right) \boldsymbol{F}_{i}
$$

Furthermore, we define $\boldsymbol{F}_{L_{i}}(i=1,2,3,4)$ as the gravitational force of Link i, and $\boldsymbol{P}_{L_{i}}(i=1,2,3,4)$ as the position of the gravitational force of Link i. Similar to Equation (1), the torque $\tau_{L_{i}}$ can be defined as

$$
\tau_{L_{i}}=\boldsymbol{S}\left(\boldsymbol{P}_{L_{i}}\right) \boldsymbol{F}_{L_{i}} .
$$

As mentioned earlier, the entire device can be divided into four parts. It is important to note that six forces are active during perching. $\boldsymbol{F}_{1}-\boldsymbol{F}_{4}$ denote the forces at the joints and $\boldsymbol{F}_{5}-\boldsymbol{F}_{6}$ denote the forces at the contact points. Each part of the device during the perching process is analyzed individually.

$$
\boldsymbol{F}_{1}+\boldsymbol{F}_{2}+\boldsymbol{F}_{L_{1}}=\mathbf{0}
$$




$$
\begin{aligned}
& \tau_{1}+\tau_{2}+\tau_{L_{1}}=0 \\
& -\boldsymbol{F}_{1}+\boldsymbol{F}_{3}+\boldsymbol{F}_{L_{2}}=\mathbf{0} \\
& -\tau_{1}+\tau_{3}+\tau_{L_{2}}=0 \\
& -\boldsymbol{F}_{3}+\boldsymbol{F}_{4}+\boldsymbol{F}_{6}+\boldsymbol{F}_{L_{3}}=\mathbf{0} \\
& -\tau_{3}+\tau_{4}+\tau_{6}+\tau_{L_{3}}=0 \\
& -\boldsymbol{F}_{2}-\boldsymbol{F}_{4}+\boldsymbol{F}_{5}+\boldsymbol{F}_{L_{4}}=\mathbf{0} \\
& -\tau_{2}-\tau_{4}+\tau_{5}+\tau_{L_{4}}=0
\end{aligned}
$$

Here, we can combine Equations (1)-(10) into the formula $A \boldsymbol{x}=\boldsymbol{b}$, where $A \in R^{12 \times 12}, \boldsymbol{x} \in R^{12}$, and $\boldsymbol{b} \in R^{12}$. The identity matrices are defined as $I$, and zero matrices and zero vectors are defined as $\boldsymbol{0}$. Specifically,

$$
\begin{aligned}
& \boldsymbol{A}=\left[\begin{array}{cccccc}
\boldsymbol{I} & \boldsymbol{I} & \mathbf{0} & \mathbf{0} & \mathbf{0} & \mathbf{0} \\
\boldsymbol{S}\left(\boldsymbol{P}_{1}\right) & \boldsymbol{S}\left(\boldsymbol{P}_{2}\right) & \mathbf{0} & \mathbf{0} & \mathbf{0} & \mathbf{0} \\
-\boldsymbol{I} & \mathbf{0} & \boldsymbol{I} & \mathbf{0} & \mathbf{0} & \mathbf{0} \\
-\boldsymbol{S}\left(\boldsymbol{P}_{1}\right) & \mathbf{0} & \boldsymbol{S}\left(\boldsymbol{P}_{3}\right) & \mathbf{0} & \mathbf{0} & \mathbf{0} \\
\mathbf{0} & \mathbf{0} & -\boldsymbol{I} & \boldsymbol{I} & \mathbf{0} & \boldsymbol{I} \\
\mathbf{0} & \mathbf{0} & -\boldsymbol{S}\left(\boldsymbol{P}_{3}\right) & \boldsymbol{S}\left(\boldsymbol{P}_{4}\right) & \mathbf{0} & \boldsymbol{S}\left(\boldsymbol{P}_{6}\right) \\
\mathbf{0} & -\boldsymbol{I} & \mathbf{0} & -\boldsymbol{I} & \boldsymbol{I} & \mathbf{0} \\
\mathbf{0} & -\boldsymbol{S}\left(\boldsymbol{P}_{2}\right) & \mathbf{0} & -\boldsymbol{S}\left(\boldsymbol{P}_{4}\right) & \boldsymbol{S}\left(\boldsymbol{P}_{5}\right) & \mathbf{0}
\end{array}\right], \\
& \boldsymbol{x}=\left[\boldsymbol{F}_{1}^{T}, \boldsymbol{F}_{2}^{T}, \boldsymbol{F}_{3}^{T}, \boldsymbol{F}_{4}^{T}, \boldsymbol{F}_{5}^{T}, \boldsymbol{F}_{6}^{T}\right]^{T} \\
& \boldsymbol{b}=-\left[\boldsymbol{F}_{\mathbf{L}_{\mathbf{1}}}{ }^{T}, \tau_{L_{1}}, \boldsymbol{F}_{\boldsymbol{L}_{\mathbf{2}}}{ }^{T}, \tau_{L_{2}}, \boldsymbol{F}_{\boldsymbol{L}_{\mathbf{3}}}{ }^{T}, \tau_{L_{3}}, \boldsymbol{F}_{\boldsymbol{L}_{\mathbf{4}}}{ }^{T}, \tau_{L_{4}}\right]^{T},
\end{aligned}
$$

Therefore, the force $\boldsymbol{x}$ can be obtained using the following equation:

$$
\boldsymbol{x}=\boldsymbol{A}^{-1} \boldsymbol{b} .
$$

\section{Classification discussion of target thickness}

As shown in Fig. 4a, we assume the ordinate distance between the lower surface of the gripper and $\boldsymbol{P}_{3}$ as $\boldsymbol{a}$ and that between the upper surface of the gripper and $\boldsymbol{P}_{\mathbf{4}}$ as $b$. Then, when the lower surface of Link 3 and the upper surface of Link 4 are parallel, we assume that the target thickness $L$ is $L_{0}$, which can be obtained as follows:

$$
L_{0}=l_{1}-a-b .
$$

As evident from Fig. 4b, c, in the cases of $L \leq L_{0}$ and $L>L_{0}$, the representations of the position vector $\boldsymbol{P}_{5}$ are different. Thus, both situations need to be

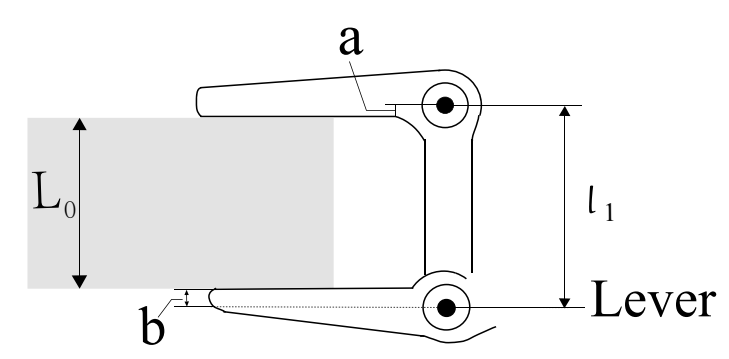

(a)

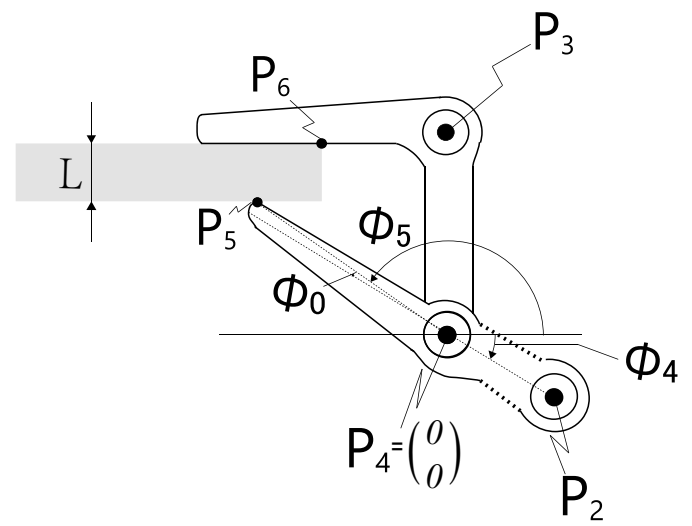

(b)

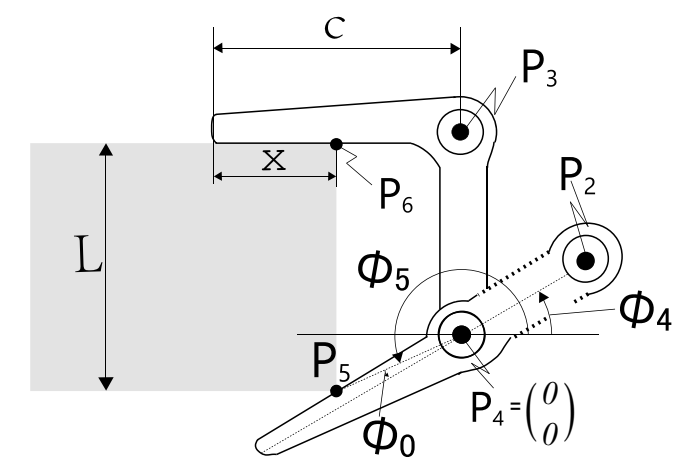

(c)

Fig. 4 a: Definition of $L_{0} ; \mathbf{b}, \mathbf{c}$ : situation analysis of $L \leq L_{0}$ and $L>L_{0}$

classified separately. Specifically, during the analysis of the perching state, we assume that the gripper of Link 3 maintains a fixed position, and we set $\boldsymbol{P}_{4}$ as the origin. Then, we can obtain the vector coordinates as follows:

$$
\boldsymbol{P}_{3}=\left[\begin{array}{l}
P_{3 x} \\
P_{3 y}
\end{array}\right]=\left[\begin{array}{l}
0 \\
l_{1}
\end{array}\right],
$$




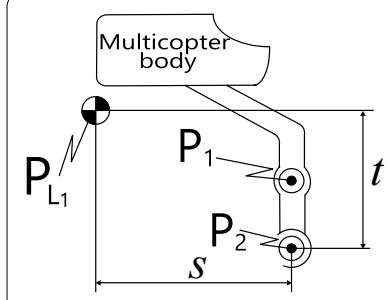

(a) COG of Link 1

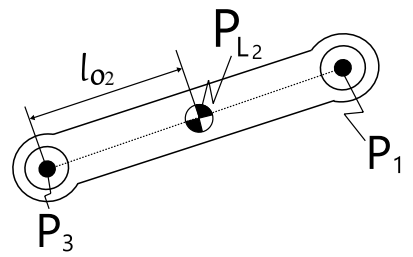

(b) COG of Link2

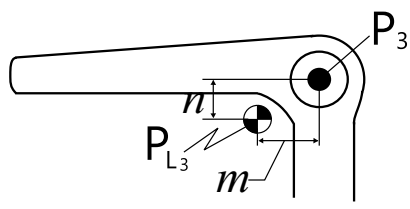

(c) COG of Link3

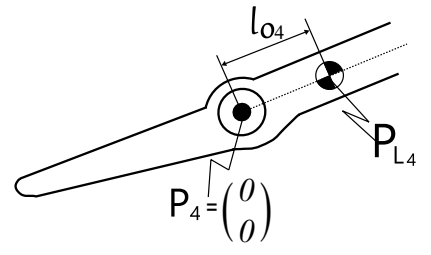

(d) COG of Link4

Fig. 5 Schematic of the center of gravity (COG) of Link 1, Link 2, Link 3, and Link 4

$$
\boldsymbol{P}_{4}=\left[\begin{array}{l}
P_{4 x} \\
P_{4 y}
\end{array}\right]=\left[\begin{array}{l}
0 \\
0
\end{array}\right] \text {. }
$$

The position vector $\boldsymbol{P}_{5}$ is at the farthest end of Link 4 . As shown in Fig. $4 \mathrm{~b}, \mathrm{c}, \phi_{5}$ is the angle between the lever and the line $\boldsymbol{P}_{4} \boldsymbol{P}_{5}$, which can be expressed as a two-variable formula:

$$
\phi_{5}=A \tan 2\left(P_{5_{y}}, P_{5_{x}}\right)
$$

Then, as shown in Fig. $4 b$, c, we assume that $\phi_{0}$ is the angle between the line $\boldsymbol{P}_{2} \boldsymbol{P}_{4}$ and the line $\boldsymbol{P}_{4} \boldsymbol{P}_{5}$, which can be obtained as follows:

$$
\phi_{0}=\sin ^{-1}\left(\frac{b}{\sqrt{\left(P_{4 x}-P_{5 x}\right)^{2}+\left(P_{4 y}-P_{5 y}\right)^{2}}}\right) .
$$

We assume that $\phi_{4}$ is the angle between the lever and the central axis of Link 4, which can be obtained as follows:

$$
\phi_{4}=\left(\phi_{5}+\phi_{0}\right)-180^{\circ} \text {. }
$$

Thus, the position vectors of $\boldsymbol{P}_{1}$ and $\boldsymbol{P}_{2}$ can be obtained as follows:

$$
\begin{aligned}
& \boldsymbol{P}_{2}=\left[\begin{array}{l}
P_{2 x} \\
P_{2 y}
\end{array}\right]=\left[\begin{array}{l}
l_{4} \cos \phi_{4} \\
l_{4} \sin \phi_{4}
\end{array}\right], \\
& \boldsymbol{P}_{1}=\left[\begin{array}{l}
P_{1 x} \\
P_{1 y}
\end{array}\right]=\left[\begin{array}{l}
P_{2 x} \\
P_{2 y}
\end{array}\right]+\left[\begin{array}{l}
0 \\
l_{1}
\end{array}\right] .
\end{aligned}
$$

(a) When thickness $L \leq L_{0}$

A situation analysis of the target thickness $L \leq L_{0}$ is shown in Fig. 4b. In the range of $0<L \leq L_{0}$, an increase in the thickness $L$ will change each position vector. We define the distance between $\boldsymbol{P}_{4}$ and $\boldsymbol{P}_{5}$ as $l_{s}$. Then, $\boldsymbol{P}_{5}$ can be obtained as follows:

$$
\boldsymbol{P}_{5}=\left[\begin{array}{l}
P_{5 x} \\
P_{5 y}
\end{array}\right]=\left[\begin{array}{l}
-l_{s} \cos \phi_{5} \\
l_{1}-a-L
\end{array}\right]
$$

(b) When thickness $L>L_{0}$

As shown in Fig. 4c, in the cases where $L>L_{0}$ the contact point $\boldsymbol{P}_{5}$ is no longer the leftmost point; thus, not only the thickness $L$ but also the embedded depth $x$ will influence the coordinates of the other position vectors. Here, we assume that $c$ is the horizontal distance between $\boldsymbol{P}_{3}$ and the tip of the upper side of Link 3 . The coordinates of the position vector $\boldsymbol{P}_{5}$ can be obtained as follows:

$$
\boldsymbol{P}_{5}=\left[\begin{array}{c}
P_{5 x} \\
P_{5 y}
\end{array}\right]=\left[\begin{array}{c}
x-c \\
l_{1}-a-L
\end{array}\right] .
$$

\section{Determination of the center of gravity of each link}

The center of gravity (COG) of each link is shown in Fig. 5. As shown in Fig. 5a, Link 1 is based on $\boldsymbol{P}_{2}$; it is offset by two parameters, namely, $s$ and $t$, in the horizontal and vertical directions, respectively. Thus, the COG of Link 1 can be obtained as follows:

$$
\boldsymbol{P}_{L_{1}}=\left[\begin{array}{l}
P_{L_{1} x} \\
P_{L_{1} y}
\end{array}\right]=\left[\begin{array}{l}
P_{2 x}+s \\
P_{2 y}+t
\end{array}\right] .
$$

Figure $5 \mathrm{~b}$ shows that Link 2 rotates based on $\boldsymbol{P}_{3}$; consequently, it can be deduced that its COG is in the middle of the line $\boldsymbol{P}_{1} \boldsymbol{P}_{3}$, and the distance between the COG and $\boldsymbol{P}_{3}$ is assumed to be $l_{o 2}$. Then, the COG of Link 2 can be obtained as follows:

$$
\boldsymbol{P}_{L_{2}}=\left[\begin{array}{l}
P_{L_{2} x} \\
P_{L_{2} y}
\end{array}\right]=\left[\begin{array}{c}
P_{3 x}+l_{o 2} \cos \phi_{4} \\
P_{3 y}+l_{o 2} \sin \phi_{4}
\end{array}\right] .
$$

Figure 5c shows that Link 3 is based on $\boldsymbol{P}_{3}$ and is offset by two parameters, namely, $m$ and $n$, in the horizontal and vertical directions, respectively. Thus, its COG can be obtained as follows:

$$
\boldsymbol{P}_{L_{3}}=\left[\begin{array}{c}
P_{L_{3} x} \\
P_{L_{3} y}
\end{array}\right]=\left[\begin{array}{c}
P_{3 x}+m \\
P_{3 y}+n
\end{array}\right]
$$


As evident from Fig. 5d, similar to Link 2, Link 4 is rotated based on $\boldsymbol{P}_{4}$ and the COG is considered to be on the central axis of Link 4 . We assume that the distance between the COG of Link 4 and $\boldsymbol{P} 4$ is $l_{04}$; the COG of Link 4 can be obtained as follows:

$$
\boldsymbol{P}_{L_{4}}=\left[\begin{array}{c}
P_{l_{4} x} \\
P_{l_{4} y}
\end{array}\right]=\left[\begin{array}{l}
P_{4 x}+l_{o 4} \cos \phi_{4} \\
P_{4 y}+l_{o 4} \sin \phi_{4}
\end{array}\right] .
$$

Specifically, the COG of the entire device is calculated by obtaining the COG of each part as follows:

$$
\boldsymbol{P}_{\text {all }}=\left[\begin{array}{c}
P_{\text {allx }} \\
P_{\text {ally }}
\end{array}\right]=\left[\begin{array}{c}
\frac{\sum_{i=1}^{4} m_{i} P_{L_{i x}}}{\sum_{i=1}^{4} m_{i}} \\
\frac{\sum_{i=1}^{4} m_{i} P_{L_{i y}}}{\sum_{i=1}^{4} m_{i}}
\end{array}\right] .
$$

\section{Classification discussion of the location of the COG}

In the previous subsection, we discussed the effect of the target factor on the perching state. Here, we will discuss the impact of the location of the COG on the perching situation. As shown in Fig. 6, when the COG of the entire device changes, the location of $\boldsymbol{P}_{6}$ is changed. Thus, when analyzing the impact of the COG, the difference in the horizontal position is an important factor, and the position where the arm is implemented depends on the parameter $s$. Here, $s$ is the most significant of all the parameters. Therefore, we assume that $s$ is a variable, whereas $t$ is constant. Consequently, the change in $s$ would change the COG of the entire device $\boldsymbol{P}_{\text {all } x}$.

\section{(a) COG is to the right of the rightmost border}

As shown in Fig. 6a, with the change in the variable $s$, the COG of the entire device is to the right of the rightmost border. We assume that the contact point of the target and the lower surface of Link 3 is located at the edge of the target. Therefore, in this case, the vector $\boldsymbol{P}_{6}$ can be obtained as follows:

$$
\boldsymbol{P}_{6}=\left[\begin{array}{l}
P_{6 x} \\
P_{6 y}
\end{array}\right]=\left[\begin{array}{l}
x-c \\
l_{1}-a
\end{array}\right] .
$$

\section{(b) COG is between the two sides of the border}

As shown in Fig. 6b, when the COG of the entire device is between both sides of the border, we assume that $\boldsymbol{P}_{6}$ has the same horizontal coordinate with the contact point of the target and the lower surface of Link 3 . Therefore, in this case, the vector $\boldsymbol{P}_{6}$ can be obtained as follows:

$$
\boldsymbol{P}_{6}=\left[\begin{array}{l}
P_{6 x} \\
P_{6 y}
\end{array}\right]=\left[\begin{array}{c}
P_{\text {allx }} \\
l_{1}-a
\end{array}\right] .
$$

\section{(c) COG is to the left of the leftmost border}

As shown in Fig. 6c, when the COG of the entire device is to the left of the leftmost border, we assume that the contact point of the target and the lower surface of Link 3 is at the edge of the gripper. Therefore, in this case, the vector $\boldsymbol{P}_{6}$ can be obtained as follows:

$$
\boldsymbol{P}_{6}=\left[\begin{array}{c}
P_{6 x} \\
P_{6 y}
\end{array}\right]=\left[\begin{array}{c}
-c \\
l_{1}-a
\end{array}\right] .
$$

\section{Categorizing and integrating various situations}

In the previous section, we separately analyzed the different factors that influence perching. However, several of these factors can occur simultaneously in practice. Thus, we need to classify and integrate various situations as shown in Fig. 7.

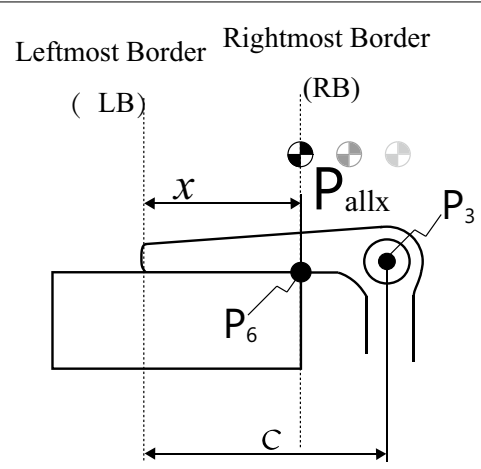

(a). COG is to the right of the rightmost border

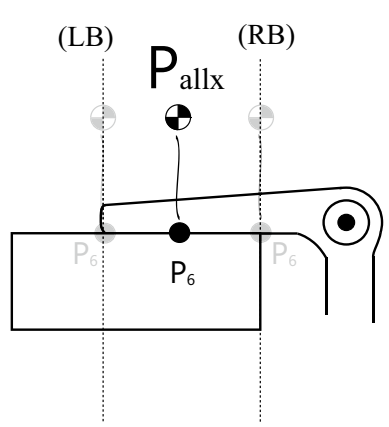

(b). COG is between the two side of the border

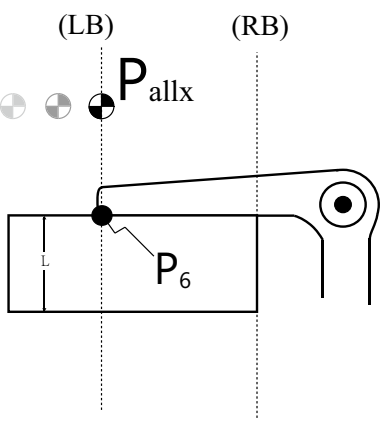

(c). $\mathrm{COG}$ is to the left of the leftmost border

Fig. 6 Factor of the position of the COG 
(Case 1) $L \leq L_{0}$, and the COG is to the right of the rightmost border.

(Case 2) $L>L_{0}$, and the COG is to the right of the rightmost border.

(Case 3) $L \leq L_{0}$, and the COG is between the two sides of the border.

(Case 4) $L>L_{0}$, and the COG is between the two sides of the border.

(Case 5) $L \leq L_{0}$, and the COG is to the left of the leftmost border.

(Case 6) $L>L_{0}$, and the COG is to the left of the leftmost border.

All the situations are analyzed and the properties of the components of the device are established. Subsequently, the forces required for perching in a certain condition can be determined using Equation (14). Based on these forces, the perching state can be assessed.

\section{Perching state judgment}

In "Available condition for stably perching" Section, we analyzed each situation related to perching. In this section, the process of judging the perching state is described. We conclude that there is an optimal range for available perching in different situations.

\section{The friction cone theory}

In this subsection, we first introduce the concept of the friction cone theory. As shown in Fig. 8a, the gripper finger contacts the target table at Point $P$, the starting point of the force. Then, we decompose the force in the horizontal and vertical directions. We assume that the horizontal component is $F$ and the vertical component is the normal vector $N$. We assume that the friction coefficient is $\mu_{s}$; thus, the maximum static friction force can be $\mu_{s} N$ or $-\mu_{s} N$. When $F$ is equal to $\mu_{S} N$, we assume that the end point of the force is Point $Q$. When $F$ is equal to $-\mu_{s} N$, we consider the end point of the force as Point $R$. Then we obtain two lines, i.e., $P Q$ and $P R$. Here, the angle between the normal vector and $P Q$ can be obtained as $\tan ^{-1} \mu_{s}$. The angle between the normal vector and $P R$ is $\tan ^{-1}\left(-\mu_{s}\right)$. We define the area inside the lines $P Q$ and $P R$ as the "friction cone."

However, if the end point of the force (Point $R$ or $Q$ ) is inside the friction cone, the value of $F$ will be lower

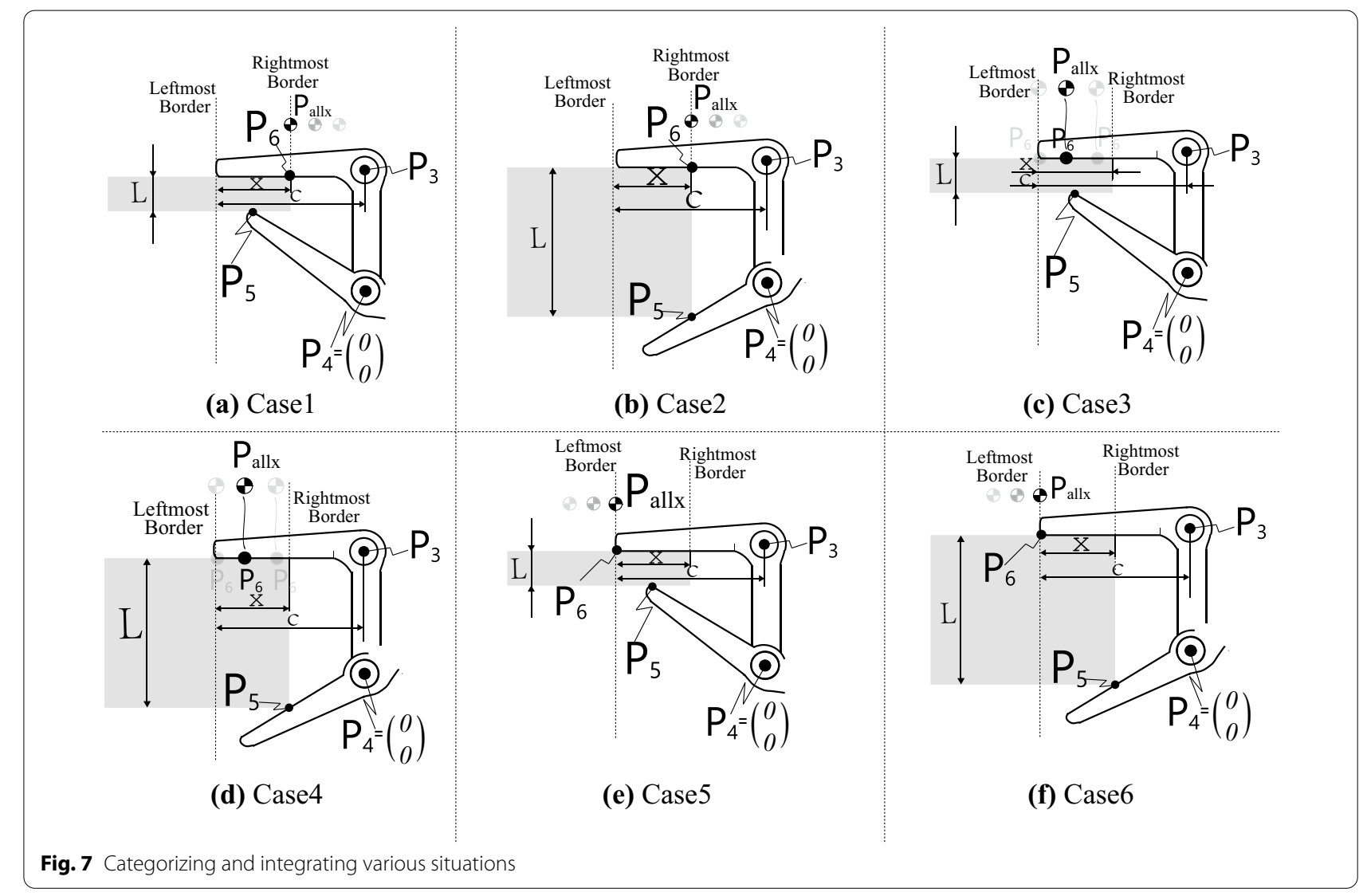




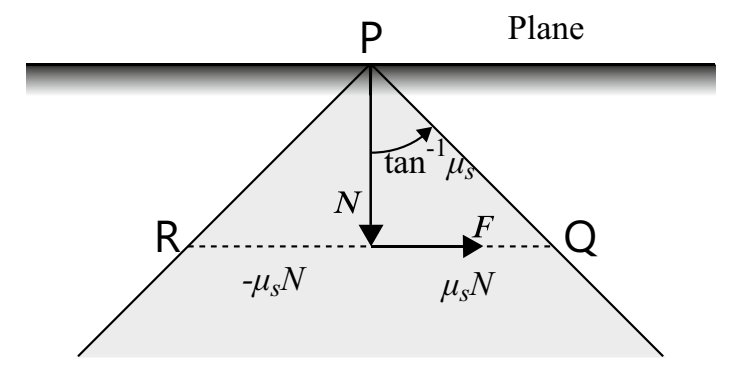

(a). Concept of the friction cone

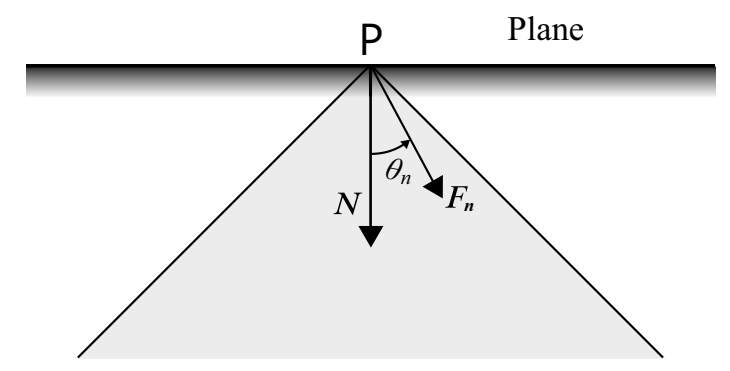

(b). Judgment by friction cone theory

Fig. 8 Concept of friction cone

than the maximum static friction force. Otherwise, the value of $F$ will be higher than the maximum static friction force. Therefore, if we describe the force, starting from $P$ as a vector, and if the end point of the vector is inside the friction cone, the gripper finger will be able to grasp the target. However, if the end point of the vector is outside the friction cone, the gripper finger will be unable to grasp the target and the perching state cannot be maintained.

\section{Judgment based on the friction cone theory}

As shown in Fig. 8b, if there is an external force $\boldsymbol{F}_{n}$ and it forms an angle $\theta_{n}$ with the normal vector $N$, the necessary condition to achieve grasping is that $\theta_{n}$ is smaller than $\tan ^{-1}\left(-\mu_{S}\right)$.

Specifically, in our study, we assumed that the angle between the grasping force and the normal vector, which starts at $\boldsymbol{P}_{5}$, was $\theta_{5}$ and that the half apex angle of the friction cone was $\theta_{\mu 5}$. Similarly, we assumed that the angle between the grasping force and the normal vector, which starts at $\boldsymbol{P}_{\mathbf{6}}$, was $\theta_{6}$ and that the half apex angle of the friction cone here was $\theta_{\mu 6}$. According to the friction cone theory, if the conditions of the angle $\theta_{5} \leq \theta_{\mu 5}$ and $\theta_{6} \leq \theta_{\mu 6}$ are satisfied, the perching state can be achieved.

The friction cone angles $\theta_{\mu 5}$ and $\theta_{\mu 6}$ can be determined through experiments. For angles $\theta_{5}$ and $\theta_{6}$, we have

$$
\begin{aligned}
& \boldsymbol{F}_{5} \cdot \boldsymbol{n}=\left\|\boldsymbol{F}_{5}\right\|\|\boldsymbol{n}\| \cos \theta_{5}, \\
& \boldsymbol{F}_{6} \cdot \boldsymbol{n}=\left\|\boldsymbol{F}_{6}\right\|\|\boldsymbol{n}\| \cos \theta_{6} .
\end{aligned}
$$

Here, $\boldsymbol{n}$ is the normal vector and $\|\boldsymbol{n}\|=1$.

Therefore, we can deduce the following:

$$
\begin{aligned}
& \theta_{5}=\cos ^{-1}\left(\frac{\boldsymbol{F}_{5} \cdot \boldsymbol{n}}{\left\|\boldsymbol{F}_{5}\right\|\|\boldsymbol{n}\|}\right), \\
& \theta_{6}=\cos ^{-1}\left(\frac{\boldsymbol{F}_{6} \cdot \boldsymbol{n}}{\left\|\boldsymbol{F}_{6}\right\|\|\boldsymbol{n}\|}\right) .
\end{aligned}
$$

Thus, we can determine whether perching space is available by comparing the relationship between $\theta_{5}$ and $\theta_{\mu 5}$, and $\theta_{6}$ and $\theta_{\mu 6}$. The simulation result is derived using the parameters in Table 1. For different $s$ values with an arbitrary target thickness $L$ and embedded depth $x$, the range of the available perching space is summarized as shown in Fig. 9. In this figure, it is difficult to show all cases of $s$ with different values. Therefore, we list the cases of $s$ in the range of -60 to $-220 \mathrm{~mm}$, in intervals of $20 \mathrm{~mm}$. In Fig. 9, the pattern of Case 5, which is shown in Fig. 7 does not appear, because, when $L \leq L_{0}$, the position of the COG is always on the right side of the leftmost border. Therefore, Case 5 is considered to be non-existent and does not appear in Fig. 9.

\section{Parallel-link passive gripper prototype and mechanism integration}

In this section, the prototype is described, particularly emphasizing the mechanism and structure of each part of the proposed perching device. The following is a description of the centering device mass, including the mechanism integration of the parts and structure.

A prototype of the proposed perching device is shown in Fig. 10. The entire device comprises two parts: a multicopter body and a perching gripper. For the multicopter body, we chose the DJI FlameWheel 550 type as the carrier of our perching device. This type of multicopter has a diagonal wheelbase of approximately $550 \mathrm{~mm}$. It is a multirotor UAV that can achieve hovering, cruising, rolling, and other flight elements. Thus, we could perform the perching experiment using this type of multicopter. The frame weighed $478 \mathrm{~g}$; the takeoff weight was approximately $1200-2400 \mathrm{~g}$, which we subsequently utilized in calculating the COG of the entire device. A 3S LiPo battery was used as the power source for the multicopter when hovering. Based on previous experimental findings, the hovering time for the DJI FlameWheel 550 with a full 3S LiPo battery can be maintained for approximately $10-12 \mathrm{~min}$. 

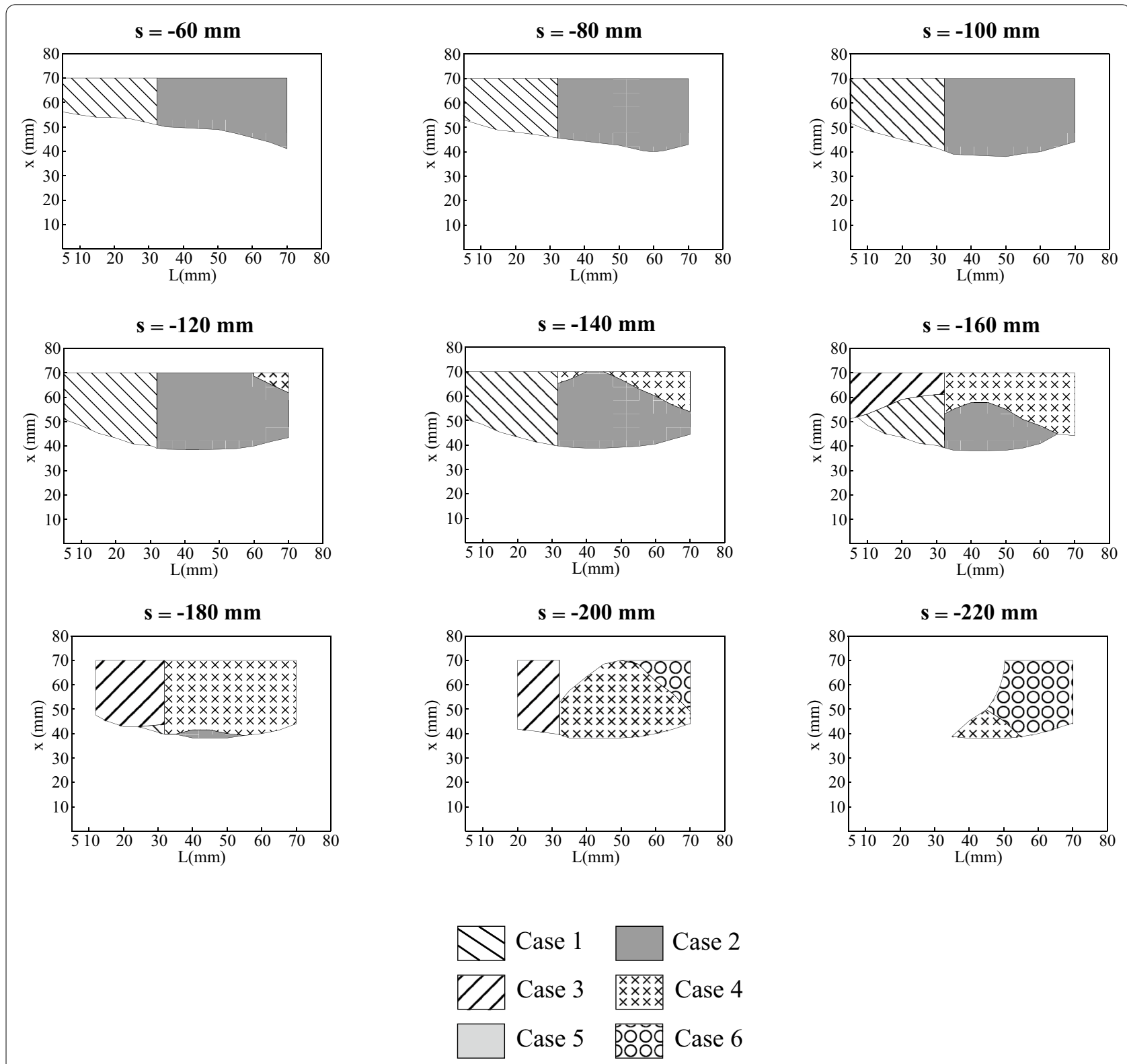

Fig. 9 Theoretical value conclusion when the value of $s$ changes

Table 1 lists the properties of the components of the entire system. Among all the situations shown in Fig. 9, the situation of " $s=-160 \mathrm{~mm}$ " contains the most cases. Therefore, we regard " $s=-160 \mathrm{~mm}$ " as the most suitable situation for conducting the experiment. Based on the analysis of the perching state as $s=-160 \mathrm{~mm}$, as stated in the previous section, we can compare the theoretical and experimental results. The experiment on the determination of possible perching is described in the following section.

\section{Experiment}

In this section, we describe an experiment on perching. In the experiment, we evaluated the possibility of perching when the gripper device was aimed at the target planes with varying thicknesses. According to the experiment, the actual plane perching conditions were implemented, and the comparison of the experimental and theoretical results is presented in this section.

In the experiment, we selected boards with thicknesses ranging from 5 to $70 \mathrm{~mm}$, and we measured the friction 


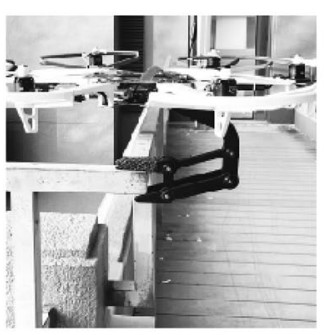

(a)

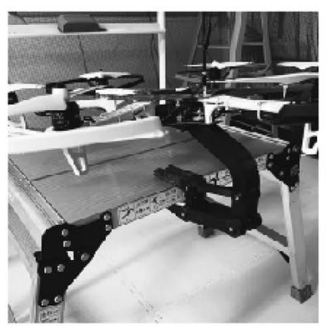

(d)

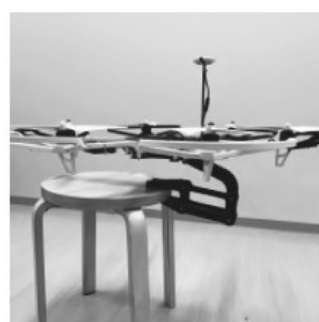

(b)

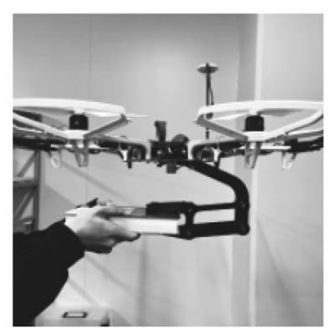

(e)

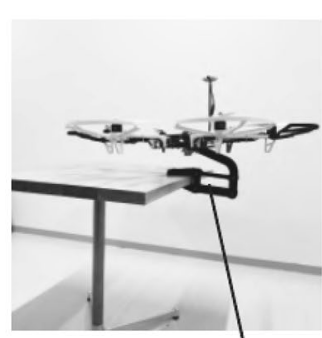

(c)

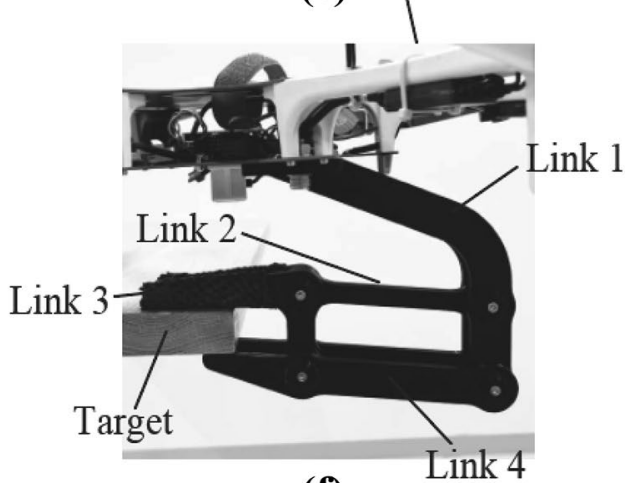

(f)

Fig. 10 Multicopter carrying the proposed device successful perches on several objects: (a) handrail, (b) chair, (c) desk, (d) ladder, and (e) a book held by a person. $(\mathbf{f})$ is the prototype of the proposed device

coefficient between the board and the gripper. Then, we obtained the values of $\theta_{\mu 5}$ and $\theta_{\mu 6}$ as $45^{\circ}$ and $40^{\circ}$ respectively. Before the experiment, we attached a spirit level to the multicopter body for judging the horizontal attitude of the multicopter. As shown in Fig. 11a, the spirit level has a bubble inside, and a circular scale is at the center of

Table 1 Properties of components of the entire system

\begin{tabular}{lll}
\hline Mass & $m_{L} 1$ & $1.738 \mathrm{~kg}$ \\
& $m_{L} 2$ & $0.022 \mathrm{~kg}$ \\
& $m_{L} 3$ & $0.025 \mathrm{~kg}$ \\
& $m_{L} 4$ & $0.025 \mathrm{~kg}$ \\
Length & $I_{1}$ & $50 \mathrm{~mm}$ \\
$I_{2}$ & $120 \mathrm{~mm}$ \\
$I_{S}$ & $61.2 \mathrm{~mm}$ \\
$I_{02}$ & $60 \mathrm{~mm}$ \\
$I_{04}$ & $21 \mathrm{~mm}$ \\
$a$ & $7.5 \mathrm{~mm}$ \\
& $b$ & $10.8 \mathrm{~mm}$ \\
& $c$ & $100 \mathrm{~mm}$ \\
Static position vector & 5 & $-160 \mathrm{~mm}$ \\
& $t$ & $96 \mathrm{~mm}$ \\
$\boldsymbol{P}_{3}$ & $(0,50)^{T}$ \\
$\boldsymbol{P}_{4}$ & $(0,0)^{T}$ \\
& $\boldsymbol{P}_{L_{3}}$ & $(-2,49)^{T}$ \\
\hline
\end{tabular}

its upper surface. The position of the bubble is changed by gradually adjusting the tilt angle, and, as shown in Fig. $11 \mathrm{~b}$, if the tilt angle is $>2.3^{\circ}$ (or $<-2.3^{\circ}$ for the opposite direction), the bubble in the spirit level will be outside the center circle scale, and this situation is considered as the multicopter cannot maintain its horizontal state. Therefore, for situations in which the bubble can be inside the center circle scale, the horizontal attitude of the multicopter can be determined. When conducting the experiment, we measured the embedded depth $x$ in millimeters to obtain the minimum and maximum limitations. The measurement was conducted from the smaller values. First, the device could not perch successfully and tilt. Subsequently, we increased $x$ in intervals of $1 \mathrm{~mm}$. When the entire device could perch successfully and maintain its horizontal posture, we recorded this value of $x$ and performed the subsequent measurement. The results are summarized in Table 2. Table 2 shows the correspondence between different cases with different plane thicknesses and different embedded depths in the perching experiment. From a comparison of the theoretical and experimental values shown in Fig. 12, the shadowed area (50\% transparency) indicates the experimental result. The common areas of the theoretical and experimental results account for a considerable proportion. The comparison in Fig. 12 demonstrates the accuracy of the analysis performed in the previous sections. 
Table 2 Experimental results

\begin{tabular}{lll}
\hline $\mathbf{L}(\mathbf{m m})$ & Min. $\mathbf{x}(\mathbf{m m})$ & Max. $\mathbf{x}(\mathbf{m m})$ \\
\hline 5 & 50 & 70 \\
10 & 48 & 70 \\
15 & 45 & 70 \\
20 & 46 & 70 \\
25 & 43 & 70 \\
30 & 41 & 70 \\
35 & 48 & 70 \\
40 & 38 & 70 \\
45 & 38 & 70 \\
50 & 37 & 70 \\
55 & 40 & 70 \\
60 & 42 & 70 \\
65 & 47 & 70 \\
70 & 56 & 70 \\
\hline
\end{tabular}

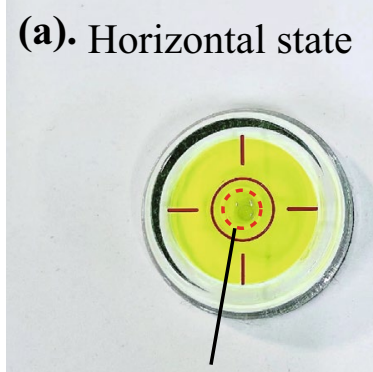

The bubble is inside

the center circle of spirit level

(Tilt angle is $0^{\circ}$ )

\section{(b). Non horizontal state}

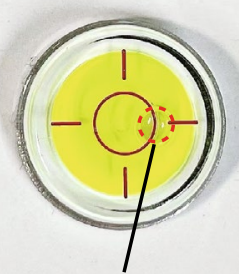

The bubble is outside

the center circle of spirit level

when tilt angle is $>2.3^{\circ}$

(or $<-2.3^{\circ}$ for the opposite direction)

Fig. 11 A spirit level is utilized to judge the horizontal attitude of the multicopter during our experiment

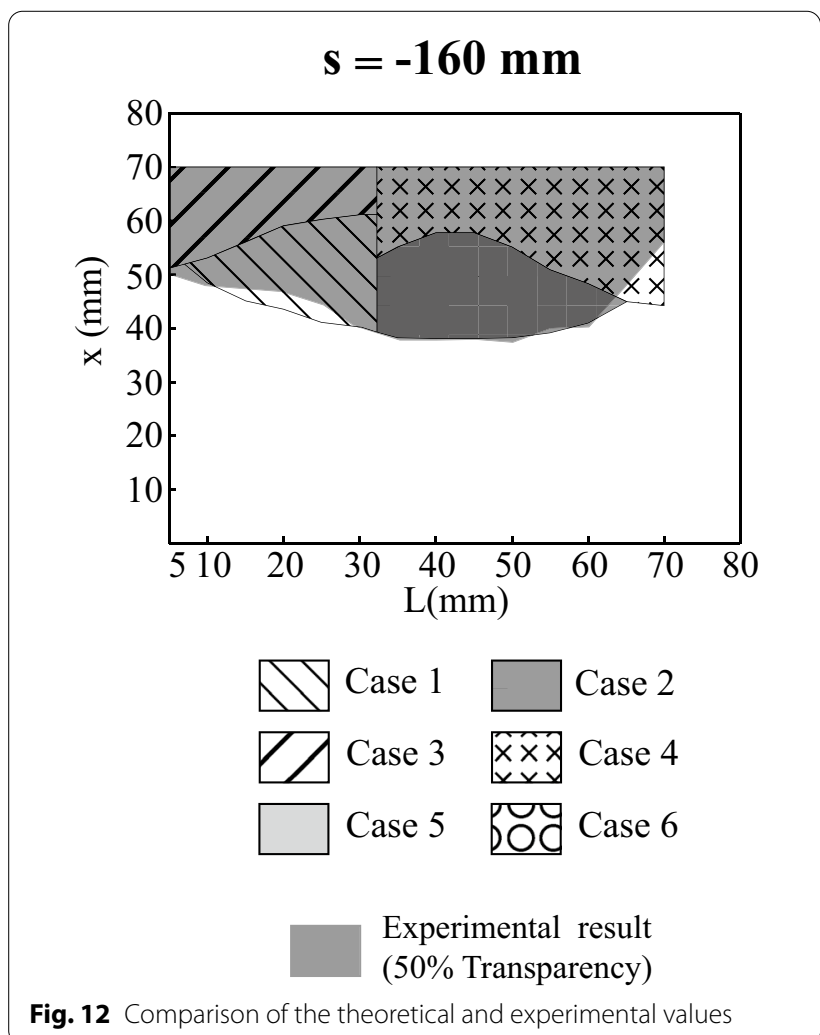

\section{Conclusion}

In this study, a multicopter carrying with a parallel-link passive gripper for available plane perching was analyzed. The judgment basis and principles were also described. The ideal condition for effective multicopter plane perching was demonstrated by comparing the theoretical and experimental results. The results indicate that there is an optimal range for possible perching for targets with varying plane thicknesses and embedded depths. However, some weaknesses and improvements also need to be considered. For example, so far, we have been unable to determine the restriction and optimal length for our gripper. Therefore, we intend to investigate this aspect in the future (Additional file 1).

Although the current study focused on perching on a plane-like target, non-horizontal targets, such as slopes or pipe-like objects, should also be considered as the target for perching. This will increase the possibility of perching in a complex environment.

\section{Supplementary Information}

The online version contains supplementary material available at https://doi. org/10.1186/s40648-022-00217-9

Additional file 1. The process of multicopter carrying with proposed gripper takeoff and perching. 


\section{Acknowledgements}

The authors would like to acknowledge the support of Smart Robotics Laboratory, Graduate School of Engineering, Hiroshima University in Japan.

\section{Authors' contributions}

MX and TT devised the concept of this study, and conducted the design and experiment. MX drafted the manuscript and TT and TS revised and refined the manuscript. All the authors have read and approved the final manuscript.

\section{Funding}

Not applicable.

\section{Availability of data and materials}

The analysis and experimental results of the current study are available from the corresponding author on reasonable request. A video file related to this study is included in the submission.

\section{Declarations}

\section{Competing interests}

The authors declare that they have no competing interests.

Received: 8 June 2021 Accepted: 2 January 2022

Published online: 01 February 2022

\section{References}

1. Patrik A, Utama G, Gunawa AAS et al (2019) GNSS-based navigation systems of autonomous drone for delivering items. J Big Data 6:53

2. Appeaning Addo K, Jayson-Quashigah PN, Codjoe SNA et al (2018) Drone as a tool for coastal flood monitoring in the Volta Delta, Ghana. Geoenviron Disasters 5:17

3. Gomez C, Purdie H (2016) UAV-based photogrammetry and geocomputing for hazards and disaster risk monitoring - a review. Geoenviron Disasters 3:23

4. Ohnishi Y, Takaki T, Aoyama T, Ishii I (2017) Development of a 4-joint 3-DOF robotic arm with anti-reaction force mechanism for a multicopter. proceeedings of 2017 IEEE/RSJ International Conference on Intelligent Robots and Systems, pp 985-991

5. Zhang Z, Xie P, Ma O (2013) Bio-inspired trajectory generation for UAV perching. IEEE/ASME International Conference on Advanced Intelligent Mechatronics. AlM, Warsaw, pp 997-1002

6. Zhang Z, Xie P, Ma O (2014) Bio-inspired trajectory generation for UAV perching movement based on tau theory. Int J Adv Robot Syst. https:// doi.org/10.5772/58898

7. Spica R, Franchi A, Oriolo G, Bülthoff HH, Giordano PR (2012) Aerial grasping of a moving target with a quadrotor UAV. 2012 IEEE/RSJ International Conference on Intelligent Robots and Systems, pp 4985-4992. https:// doi.org/10.1109/IROS.2012.6385771

8. Crisman JD, Kanojia C, Zeid I (1996) Graspar: a flexible, easily controllable robotic hand. IEEE Robot Autom Mag 3(2):32-38. https://doi.org/10.1109/ 100.511778

9. Almasri B, Ouezdou FB (2007) New design of one motor driven under actuated humanoid hand. 2007 IEEE/RSJ International Conference on Intelligent Robots and Systems, San Diego, pp 1491-1496. https://doi. org/10.1109/IROS.2007.4399057

10. Massa B, Roccella S, Carrozza MC, Dario P (2002) Design and development of an underactuated prosthetic hand. Proc 200 IEEE Int Conf Robot Autom 4:3374-3379. https://doi.org/10.1109/ROBOT.2002.1014232

11. Nagendran A, Crowther W, Richardson R (2012) Biologically inspired legs for UAV perched landing. IEEE Aerosp Electron Syst Mag 27(2):4-13

12. Chi W, Low KH, Hoon KH, Tang J, Go TH (2012) A bio-inspired adaptive perching mechanism for unmanned aerial vehicles. J Robot Mechatronics 24(4):642-s648

13. Wopereis HW, van der Molen TD, Post TH, Stramigioli S, Fumagalli M (2016) Mechanism for perching on smooth surfaces using aerial impacts. 2016 IEEE International Symposium on Safety, Security, and Rescue Robotics (SSRR), Lausanne, pp 154-159. https://doi.org/10.1109/SSRR. 2016.7784292
14. Doyle CE et al (2013) An avian-inspired passive mechanism for quadrotor perching. IEEE ASME Trans Mechatronics 18(2):506-517. https://doi.org/ 10.1109/TMECH.2012.2211081

15. Doyle CE et al (2011) Avian-inspired passive perching mechanism for robotic rotorcraft. 2011 IEEE/RSJ International Conference on Intelligent Robots and Systems, San Francisco, pp 4975-4980. https://doi.org/10. 1109/IROS.2011.6094487

16. Xu M, Takaki T, Jiang M, Ishii I (2019) Development of parallel-link-passivegripper by using a multicopter's own weight for perching. Proceedings of the SICE Annual Conference 2019, Hiroshima, pp 431-432

\section{Publisher's Note}

Springer Nature remains neutral with regard to jurisdictional claims in published maps and institutional affiliations.

\section{Submit your manuscript to a SpringerOpen ${ }^{\circ}$ journal and benefit from:}

- Convenient online submission

- Rigorous peer review

- Open access: articles freely available online

- High visibility within the field

- Retaining the copyright to your article

Submit your next manuscript at $\boldsymbol{\nabla}$ springeropen.com 